\section{G119(P) PATIENTSAFETY INCIDENT REPORTING DATA TRENDS OF A REGIONAL NEONATAL TRANSFER SERVICE}

${ }^{1} \mathrm{SL}$ Davidson, ${ }^{1,2} \mathrm{~N}$ Ratnavel, ${ }^{1,2,3} \mathrm{~A}$ Sinha, ${ }^{1,2} \mathrm{~S}$ Mohinuddin. 'London Neonatal Transfer Service, Bart's Health NHS Trust, London, UK; ${ }^{2}$ Neonatal Unit of the Children's Hospital Based at the Royal London Hospital, Bart's Health NHS Trust, London, UK; ${ }^{3}$ Centre for Paediatrics, Blizard Institute, Barts and the London School of Medicine and Dentistry, London, UK

\subsection{6/archdischild-2015-308599.118}

Introduction Neonatal transfers take place in a high-risk environment and patient safety must be a key priority.

Methods Risk reporting for this regional transfer service has evolved over the last five years. The patient safety incident form has been adapted over the last 3 years, providing a clear structure of what is to be reported, ensuring the significance of the adverse event is considered and the response to the incident recorded. This study reviewed the patient safety incident forms completed over the last three years.

Results Form completion significantly improved from $61 \%$ in 2011 to $96 \%$ in 2013. Comparing trends between 2012 and 2013: significant improvements were seen in delayed dispatch to time critical transfers, time delays during stabilisation, equipment problems, vascular incidents and unintended hypothermia. However, a significant increase in hypocarbia and endotracheal tube repositioning.Conclusion We report improved risk reporting and an improvement in many of the incident categories. Future work needs to focus on sustaining and improving other categories.

\section{G120(P) FOOD PROTEIN INDUCED ENTEROCOLITIS SYNDROME (FPIES) IS AN IMPORTANT DIFFERENTIAL DIAGNOSIS OF NECROTIZING ENTEROCOLITIS IN PRETERM INFANTS}

${ }^{1} \mathrm{M}$ Nash, ${ }^{1} \mathrm{~A}$ Bedford Russell, ${ }^{1} \mathrm{G}$ Holder, ${ }^{1} \mathrm{~A}$ Singh, ${ }^{2} \mathrm{~S}$ Murch. ${ }^{1}$ Neonatal Unit, Birmingham Women's Hospital, Birmingham, UK; ${ }^{2}$ Warwick Medical School, University of Warwick, Warwick, UK

\subsection{6/archdischild-2015-308599.119}

Aims GI dysmotility is common in preterm infants. Necrotizing enterocolitis (NEC) may develop when dysmotility, luminal contents and gut bacteria drive inflammation. Breast feeding is notably protective. Food Protein Induced Enterocolitis Syndrome (FPIES) may mimic NEC, including distension and intramural gas. In contrast to NEC, FPIES manifests thrombocytosis and neutrophilia. We have examined whether cases initially diagnosed as NEC were due to FPIES induced by cow's milk (CM) protein.

Methods 9 preterm infants from one tertiary centre (23-36 weeks, BW 535-1700 g) were identified as possible FPIES because of temporal link between introduction of CM and onset of acute GI symptoms. A timeline was obtained of symptoms, feeding and blood parameters.

Results 3/9 showed gastro-oesophageal reflux \pm constipation, improving on breast milk exclusion and worsening on reintroduction. NEC was queried but not diagnosed. All gained weight poorly, leading to CM formula introduction, which substantially worsened symptoms. All settled on exclusion and relapsed on CM challenge, remitting only with hypoallergenic formulae. Investigations showed thrombocytosis $(>400)$ and reduced albumin after CM introduction. 6/9 were diagnosed with NEC, two twice. All showed similar dysmotility and poor weight gain on breast milk, and in all cases NEC symptoms began within $48 \mathrm{~h}$ of introduction of $\mathrm{CM}$ formula \pm fortifier or thickener. In $1 /$ 6 thrombocytopaenia and neutropaenia was consistent with classic NEC, while in 5/6 platelets increased (mean 422, range 310-550) as did wcc (mean 17, range 16-25). All settled only on hydrolysate or amino acid formula.

Conclusion The NEC-like episodes in these infants concord with classic reports of FPIES in LBW infants. Most documented cases of NEC develop thrombocytopaenia - indeed thrombocytosis is unreported. All showed dysmotility, poor weight gain and thrombocytosis on breast milk: a pattern characteristic of nonIgE-mediated allergy. CM introduction because of poor weight gain was uniformly deleterious. Recognition that non-IgE-mediated CM allergy may cause dysmotility and impaired growth in preterm infants should promote consideration of maternal milk exclusion diets while breastfeeding, or introduction of hypoallergenic rather than CM formulae. Thrombocytosis in a preterm infant with dysmotility should be a red-flag sign for non-IgEmediated food allergy and risk of FPIES on formula introduction.

\section{G121(P) SERVICE EVALUATION OF MANAGEMENT OF TERM NEONATES WITH MAJOR PERINATAL BLOOD LOSS}

R Jones, J Sayge, D Evans. Neonatal Intensive Care Unit, North Bristol NHS Trust, Southmead Hospital, Bristol, UK

10.1136/archdischild-2015-308599.120

\begin{tabular}{|c|c|c|c|c|c|c|c|}
\hline Year & 2011 & & 2012 & & 2013 & & Significance \\
\hline Filled Forms /Total Transfers & $812 / 1338$ & & $1027 / 1389$ & & $1321 / 1374$ & & $\mathrm{p}<0.001$ \\
\hline \multirow[t]{2}{*}{ Incidents reported } & 283 & & 471 & & 465 & & \\
\hline & No (\%forms) & $\%$ Transfers & No (\%forms) & $\%$ Transfers & No (\% forms) & $\%$ Transfers & Comparing 2012-13 \\
\hline Dispatch $>60 \mathrm{~min}^{*}$ & $18(6.4)$ & 1.3 & $53(5.2)$ & 3.8 & $32(2.4)$ & 2.3 & $p<0.001$ \\
\hline Time Delays & $77(27.2)$ & 5.8 & $163(34.6)$ & 11.7 & $6(1.3)$ & 0.4 & $\mathrm{p}<0.001$ \\
\hline Vehicle/Equipment Problem & $57(20.1)$ & 4.3 & $53(11.3)$ & 3.8 & $37(8)$ & 2.7 & $p=0.003$ \\
\hline Medication Error & $4(1.4)$ & 0.3 & $3(0.6)$ & 0.2 & $9(1.9)$ & 0.7 & $p=0.19$ \\
\hline Hypocarbia $\left(\mathrm{pCO}_{2}<4 \mathrm{kPa}\right)$ & $27(9.5)$ & 2 & $67(14.2)$ & 4.8 & $122(26)$ & 8.9 & $p=0.017$ \\
\hline ET repositioned $(<\mathrm{T} 1$ or $>\mathrm{T} 2)$ & $33(11.7)$ & 2.5 & $50(10.6)$ & 3.6 & $118(25.4)$ & 8.6 & $\mathrm{p}<0.001$ \\
\hline Vascular access related & $29(10.2)$ & 2.2 & $42(8.9)$ & 3 & $9(1.9)$ & 0.7 & $\mathrm{p}<0.001$ \\
\hline Loss of ET tube/catheter & $6(2.1)$ & 0.4 & $7(1.5)$ & 0.5 & $15(3.2)$ & 1.1 & $p=0.257$ \\
\hline Hypothermia $(<36.5)$ ** & $53(18.7)$ & 4 & $121(25.7)$ & 8.7 & $70(15.1)$ & 5.1 & $p<0.001$ \\
\hline Communication issues & $1(0.4)$ & 0.1 & $32(6.8)$ & 2.3 & $52(11.2)$ & 3.8 & $p=0.288$ \\
\hline
\end{tabular}

*National Time Critical Category **Unintended 
Aims Clear guidelines exist for the management of major haemorrhage in adults. These include a strong emphasis on early transfusion of Fresh Frozen Plasma (FFP) in conjunction with Packed Red Cells (PRC) in order to avoid/treat possible accompanying coagulopathy. Current practice within the local neonatal population is to await clotting profiles and administer FFP if coagulation is found to be abnormal. This practice may lead to a significant delay in treatment. The aim of this service evaulation was to identify whether term neonates with major haemorrhage around the time of delivery required support with coagulation factors. This may support the use of prophylactic FFP alongside $\mathrm{RBC}$ transfusion prior to results becoming available helping to stabilise the infant sooner.

Methods Data was requested from our local blood transfusion laboratory for all requests for blood products made for patients on the neonatal unit between April 2011 to December 2013. Further details regarding antenatal and neonatal course were then taken from the Badger database on all babies that were identified to have received blood products on their date of birth or following day. Babies born at less than 37 weeks were excluded.

Results We identified 7 term babies with a clear history of major perinatal blood loss. $5 / 7$ (71\%) required transfusion of FFP in addition to PRC on the basis of abnormal clotting profile. All babies receiving FFP needed extensive resuscitation at birth followed by intensive care support, whereas the 2 babies that were not transfused were clinically stable. One of the babies not receiving FFP did not have their clotting profile checked. All babies received Vitamin $\mathrm{K}$ post delivery as standard.

Conclusion Term infants with a clear history of perinatal blood loss who are compromised at delivery and require packed red cell transfusion should be considered for early transfusion of FFP without awaiting coagulation profiles.

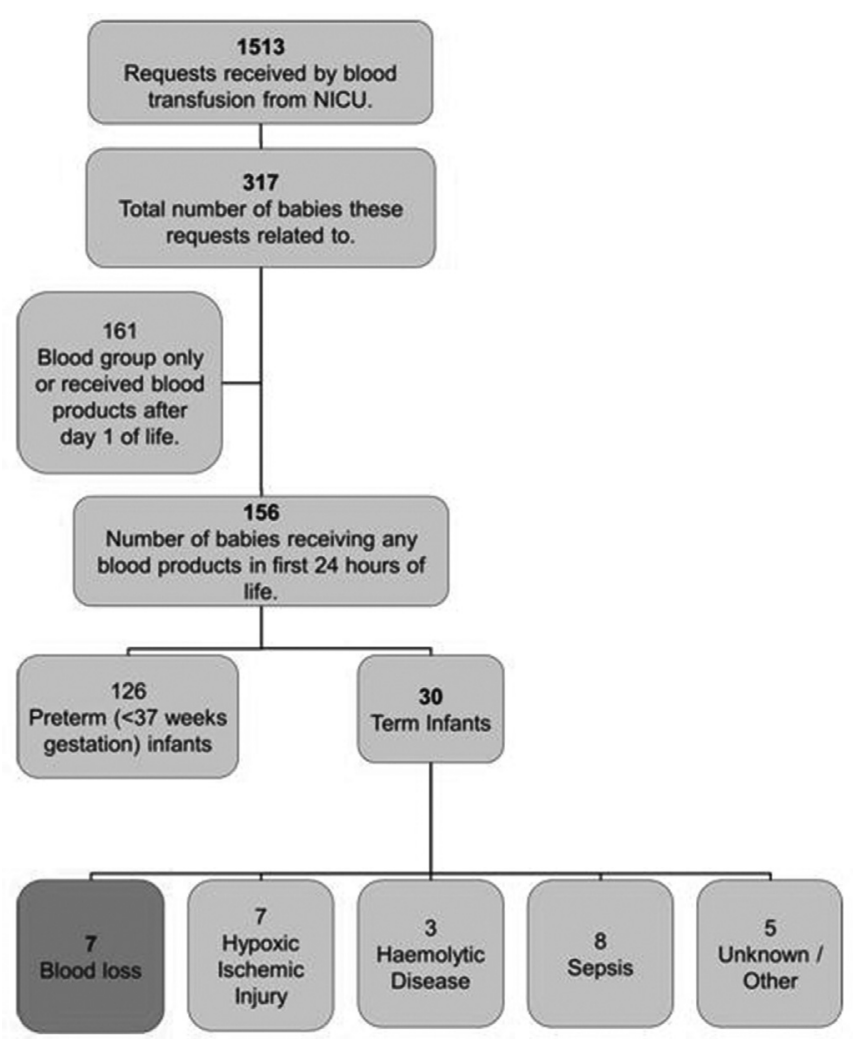

Abstract G121(P) Figure 1 Participant flow diagram

\section{G122(P) MELATONIN AND IMMUNE CELL RESPONSES IN NEONATAL ENCEPHALOPATHY}

${ }^{1} S$ Asalm, ${ }^{2}$ RWG Watson, ${ }^{2}$ A O'Neill, 3,4,5EJ Molloy. ${ }^{1}$ Neonatology, National Maternity Hospital, Holles Street, Dublin, Ireland; ${ }^{2}$ Conway Institute for Biomolecular and Biomedical Science, University College Dublin, Dublin, Ireland; ${ }^{3}$ Neonatology, Our Lady's Children's Hospital, Crumlin, Ireland; ${ }^{4}$ Academic Paediatrics, Trinity College Dublin, National Children's Hospital, Tallaght, Ireland; 'PPaediatrics, Coombe Women's and Infant's University Hospital, Dublin, Ireland

\subsection{6/archdischild-2015-308599.121}

Introduction Infection and inflammation can be antecedents of Neonatal Encephalopathy (NE) and increase the risk of neurological sequelae. Melatonin is a potent immunomodulator and antioxidant (1) and may alter the systemic inflammatory response in NE (2).

Aim To investigate the in vitro effect of melatoninon whole blood reactive oxygen intermediates (ROI), CD11b and Toll-like receptor (TLR)-4 in neutrophils and monocytes from infants with NE receiving therapeutic hypothermia (TH) versus healthy neonatal controls in the first week of life.

Methods Infants with NE were recruited and their demographics details, grade of NE, MRI results, outcome and placental histology were recorded. Whole blood was taken on Day 1,3 and 7 of life (NE group) and day 1(Controls) and flow cytometry used to assess TLR4, CD11b and ROI in both monocytes and neutrophils in the presence of Lipopolysaccharide (LPS) and/or melatonin. Ethics was received from ethics committee at National Maternity Hospital.

Results LPS-induced ROI production was significantly increased in both neutrophils and monocytes $(\mathrm{p}=0.03)$ in NE versus controls $(n=6)$ on day 1 of life. On day 7 of life, following $\mathrm{TH}$, LPS-induced CD11b upregulation was significantly decreased by melatonin in vitro in neonates with NE $(n=7)$. There was no difference in TLR4 expression in NE and controls.

Conclusion Melatonin decreases the production of CD11b in neutrophils, which is a marker of neutrophil activation and migration and may ameliorate the augmented systemic inflammatory response seen in infants with NE.

\section{G123(P) SURVEY OF DELIVERY ROOM PRACTICE: RESUSCITATION OF EXTREME PRETERM INFANTS}

S Al-Jilaihawi, A Huertas-Ceballos. Neonatal Department, University College Hospital, London, UK

\subsection{6/archdischild-2015-308599.122}

Aims There are Nuffield Guidelines in place to aid clinicians in their decision-making in the resuscitation of extreme preterm infants. This remains an area where there is varied practice nationally and internationally, and decisions are often case dependant. We sought to survey a cohort of Consultant Paediatricians involved in neonatal resuscitation on their approach to the resuscitation of extreme preterm infants, to assess current attitudes to practice.

Methods A questionnaire was designed using an online survey programme, modelled on the Nuffield guidance for resuscitation of extreme preterm infants. This was distributed to a group of Consultants via email and results were collated using the online programme.

Results 45 of 68 (66\%) Consultants completed the survey. 26\% of responders were from level 3 neonatal intensive care units (NICU), 62\% from level 2 units and 12\% from level 1 units. 